\title{
Connected Wireless Camera Network Deployment with Visibility Coverage
}

\author{
Hua Huang* Chien-Chun $\mathrm{Ni}^{\dagger} \quad$ Xiaomeng $\mathrm{Ban}^{\dagger} \quad \mathrm{Jie}^{\dagger} \mathrm{Gao}^{\dagger} \quad$ Andrew T. Schneider* Shan Lin* \\ * Department of Computer and Information Science, Temple University. \{hua.huang, andrew.thomas.schneider, shan.lin\}@temple.edu \\ $\dagger$ Department of Computer Science, Stony Brook University. \{chni, jgao, xban\}@cs.stonybrook.edu
}

\begin{abstract}
We consider the problem of deployment of cameras inside a complex indoor setting for surveillance applications. We formulate the problem of the minimum guarding network that places a minimum number of cameras satisfying both visual coverage of the domain and wireless network connectivity. We prove that finding the minimum guarding network in both the geometric setting and discrete setting is NP-hard. We also give a 2-approximation algorithm to the geometric minimum guarding network. Motivated by the connection of this problem with the watchman tour problem and the art gallery problem, we develop two algorithms that generate satisfactory results in a prototype testbed and in our simulations.
\end{abstract}

Index Terms-Visibility Coverage, Wireless Connectivity, Camera Networks

\section{INTRODUCTION}

With the recent advancement of pervasive computing, wireless networks, and optical sensing, wireless camera networks are deployed for a wide range of applications. In many existing systems for infrastructure monitoring and home healthcare, camera network deployments heavily rely on existing infrastructure. Typically, a node is plugged into the wall and directly connects to a local network access point through wire or wireless. However, infrastructure support is not available in many scenarios such as first responder and military applications, where the network needs to be deployed quickly with little infrastructure support. For example, soldiers deploy a camera network to monitor suspicious activities in a building during military operations. These applications impose a unique set of requirements on camera network deployment: (1) Full visibility coverage: the whole building needs to be monitored completely; (2) Wireless connectivity: all camera nodes need to be self-organized to form a wireless network to transfer data to a base station over wireless communications. Relay nodes may be introduced. (3) Low deployment cost: it is desirable to use a minimum number of devices for the low deployment effort and short deployment time. In addition, the floor plan of the interior of a building typically has a complex geometric shape. All these constraints make the camera network deployment problem very challenging.

In this paper, we initiate the study of camera deployment that satisfies both visibility coverage and wireless connectivity. For modeling visibility coverage, we assume that each camera's sensing range is only restricted by line of sight. This is a generalization of the "cone" model [32] of a camera. To enable wireless communication, we assume that wireless 978-1-4799-3360-0/14/\$31.00 (C)2014 IEEE camera nodes and relay nodes have fixed communication range of $r$. When the cameras are deployed such that they do not naturally form a connected network, wireless relay nodes may be introduced to restore connectivity. With the two models, we formulate the Minimum Connected Guarding Network Problem, which minimizes the number of cameras and relay nodes while satisfying both visibility coverage and wireless connectivity constraints.

The integration of isotropic sensing and wireless networking makes this problem unique and interesting. Many previous studies have proposed algorithms $[9,25,31]$ to maximize the visibility coverage of the camera network, but little attention is given to the problem of providing a connected network while minimizing the wireless communication cost, which is an essential requirement under many applications. Whereas related sensor network coverage research $[14,15]$ provides valuable insights to sensor deployment, they usually assume short range circular sensing models, which do not apply for optical cameras. This paper will focus on filling this missing gap.

The solution to the minimum connected guarding network problem clearly depends on the scale of the wireless communication range of the sensor nodes. Take one extreme, say the communication range is large enough such that any two nodes inside the building can directly communicate with each other. this problem boils down to the classical Art Gallery Problem (AGP), which aims at finding a minimum number of guards such that any point in the building is within direct line of sight of at least one guard. The art gallery problem is a well known NP-hard problem and a considerable amount of literature has been published on this topic[17][12][24]. Take the other extreme, say that the communication range is very small compared to the scale of the area being monitored, we basically need to place the sensors along a path to keep them connected. Thus the problem converges to finding a connected geometric network such that any point in the area is visible to at least one point of the network. It is not hard to show that such a network must be a tree when its length is minimized. So far, however, there has been little discussion about finding a minimum guarding tree for a polygon. A closely related problem is the watchman route problem [2], i.e., finding a route of minimum length that guards an entire polygon. It is known that the watchman route problem is NP-hard for the general polygon with holes [5]. But nothing is known about 
the hardness if the problem if the nodes are connected as a tree.

In this paper we first show that the Minimum Connected Guarding Network problem is NP-hard, when the wireless communication range is a constant $r$. We also show that under one extreme setting that the communication range converges to zero, this problem, which is termed the Geometric Minimum Guarding Network problem, is still NP-hard to solve. We then give an approximation algorithm with constant approximation ratio to this special case [2].

In terms of algorithm development for practical implementations, we consider two possible deployment settings. In the first setting, termed the Connected Visibility region Planning (CVP), we assume that the building floor plan is known and the network designer has plenty of time to deploy and optimize the network. CVP first identifies a set of camera locations that guarantee the visibility coverage of the domain, and then remove redundant cameras by identifying overlapping viability regions. This algorithm is computationally intensive but produces near optimal deployment result in practice. In the second setting, termed the Connected Visibility region Tracking (CVT), we assume first responder applications in which in-field deployment must be done quickly. We employ the watchman tour based solution, which places sensors along the shortest watchman route.

The proposed deployment algorithms are evaluated in real scenarios. We build a wireless camera network testbed to validate the effectiveness and accuracy of our algorithms. Each node of this testbed consists of four off-the-shelf components: a beagle board computer, a $\mathrm{Wi}-\mathrm{Fi}$ radio, a webcam, and a battery cape. Real deployment in our campus building achieves above $99 \%$ wireless connectivity and satisfactory visibility coverage (Currently the cameras are not panoramic). We also implement a simulation framework to compare the performance of CVP, CVT and the classical 3-coloring algorithm. Compared with existing solutions, our deployments strategies have significantly better wireless communication connectivity and lower power consumption.

\section{MODEL AND HARDNESS}

In this section, we will formulate the wireless camera deployment problem rigorously and prove the hardness of the problem.

We consider an indoor scenario and the domain of deployment is modelled by a polygon $P$. A camera node is a wireless node with visual sensing range defined by line of sight, and wireless communication range defined as a disk of radius $r$. We would like to place a minimum number of cameras inside $P$ such that two conditions are met:

- All nodes collectively "guard" the entire polygon $P$ in the sense that any point of $P$ has a direct line of sight path to at least one camera;

- The nodes form a connected network using wireless communication.

This problem is denoted as the Minimum Connected Guarding Network problem.
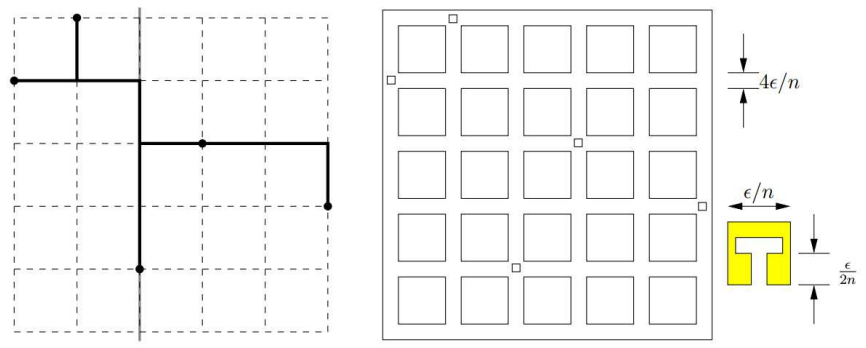

Fig. 1: Given an instance of the minimum geometric rectilinear Steiner tree (left), we turn it to an instance in which each point is replaced by a T-junction obstacle (shown to the right).

If the input polygon is convex, then the camera deployment problem is trivial - placing one camera in any location inside the polygon can ensure full coverage (and it is trivially a connected network). Therefore, we will focus on the setting when $P$ is non-convex.

Theorem 1. Finding the minimum connected guarding network is NP-hard, even in a simple polygon $P$.

Proof: We use an induction from the standard art gallery problem. Given an arbitrary art gallery instance with an input polygon $P$, we scale the polygon $P$ down such that it is within a unit disk. The optimum solution for the art gallery problem does not change. But the cameras in any guarding solution form a complete graph. Thus if we have a solution for the minimum connected guarding network, it is the optimal solution for the art gallery problem, which is known to be NPhard to find.

Notice that the proof above depends on the communication range being a fixed constant. When the size of the deployment domain is much greater than the communication range, i.e., $r \rightarrow 0$, the minimum connected network becomes a geometric graph that guards the polygon $P$. We would like to find such a geometric network with minimum total length. We call this problem the Minimum Geometric Guarding Network problem. This problem is also NP-hard for a general polygon $P$.

Theorem 2. Finding the minimum geometric guarding network in a general polygon with holes is NP-hard.

Proof: We use reduction from the minimum geometric rectilinear Steiner tree problem in the plane. Given $n$ points on a unit lattice called sites, we would like to find a tree $T$ connecting the $n$ sites with minimum total length. The tree may use other non-site lattice points as vertices and all edges of the tree must be either horizontal or vertical. See the left figure in Figure 1. Given such an instance, we construct an instance for the guarding problem. We first enlarge lattice edges to narrow corridors. Each lattice grid becomes a 'hole' of the polygon. In particular, a site vertex will map to a small 'T-junction' gadget hole such that one must visit the junction point in order to guard it. The T-junction hole is small enough to fit inside the corridor. See Figure 1 for the sizes of the corridor and the T-junction hole.

Now we can verify that for a positive integer $m$, there exists 
a rectilinear Steiner tree of length at most $m$ if and only if there exists a minimum geometric guarding network of length at most $m+3 \epsilon$. Take a very small $\epsilon$ say 0.1 . This shows that the minimum geometric guarding network problem is NP-hard for a polygon with holes.

Given the hardness results, we then move on to find approximation algorithms and practical solutions with good performance in reality.

\section{Minimum Geometric Guarding Network}

In this section we first show some useful properties of the minimum geometric guarding network. Then we present a 2-approximate solution for the minimum geometric guarding network problem in a simple polygon. This algorithm is also a building block for the general case when $r \neq 0$, to be discussed afterwards.

We represent the input polygon $P$ by a sequence of vertices $v_{1}, v_{2}, \cdots, v_{n}$, with $n \geq 4$. For $i=1,2, \ldots, n-1$, $e_{i}=\left(v_{i}, v_{i+1}\right)$ represents an edge of the polygon connecting node $v_{i}$ and $v_{i+1}$. For ease of presentation, we also impose a direction upon each edge such that the interior of the polygon lies to the left of the edge, or equivalently, the boundary of $P$ is directed counterclockwise. We also assume that without loss of generality that the vertices of $P$ are in general position, i.e., no three vertices are collinear.

A vertex $v$ is a reflex vertex if the interior angle at $v$ is greater than $\pi$; a vertex is called convex otherwise. A chain of vertices between $v_{i}$ and $v_{j}$ is defined as all the vertices that will be encountered if one scans from $v_{i}$ counterclockwise to $v_{j}$. The visibility polygon of a point $x$ inside $P$, denoted by $V(x)$, is defined as the set of points in $P$ with direct line of sight from $x$.

We say a set of points $M$ inside $P$ are guards or a guard cover, if for any point $p \in P$, there is a point $q \in M$ such that $q$ sees $p$. We also say that a guard cover is able to guard $P$.

Theorem 3. Given a polygon $P$, the minimum geometric network is a tree of polygonal curves.

Proof: Take any geometric guarding network $G$ within $P$, we can find a finite size guard cover $M$ on $G$. In particular, we take each reflex vertex $v_{i}$ and extend its two adjacent edges, $v_{i-1} v_{i}$ and $v_{i} v_{i+1}$. This gives us at most $n$ lines forming an arrangement cutting the polygon $P$ into convex pieces. We take all vertices of this arrangement within $P$. For each such vertex, we take a point on $G$ visible to it and add it to the guard cover $M$. Clearly the number of guards is at most $O\left(n^{2}\right)$. Further, the points of $M$ is a guard cover.

Now take a minimum Steiner tree $T$ upon the guards $M$. Clearly $T$ guards $P$. Also $T$ is no longer than the total length of $G$. This shows that the minimum geometric guarding network must be a tree made of polygonal curves.

Therefore from the above theorem we can also denote the optimal solution as the minimum geometric guarding tree.

To get a 2-approximation to the minimum geometric guard network, the idea is to make use of a watchman tour for a given polygon $P$. A watchman tour is a (closed) cycle inside $P$ that guards $P$. That is, any point of $P$ has direct line of sight to at least one point on the tour [3]. Although finding the shortest watchman tour in a general polygon with holes is NP-hard [3], there is an $O(\log n)$-approximation algorithm for a rectilinear version with restricted visibility [20]. The watchman tour problem for a simple polygon is solvable in polynomial time (for a tour with a fixed starting point see [26, 29], and for the floating tour without a given starting point see [27]).

We show in the following theorem that the optimum watchman tour is a 2-approximation to the minimum geometric guarding network.

Theorem 4. Inside a polygon $P$, the optimum watchman tour is a 2-approximation to the minimum geometric guarding network. This is true for both cases when a fixed starting point is given, or not given.

Proof: First any watchman tour is clearly a geometric guarding tree. We take the minimum geometric guarding tree $T$, double all edges in the tree which then form a tour along the tree, visiting each edge exactly twice, once in each direction. This resulting tour is a watchman tour. It has length exactly twice the length of the minimum geometric guarding tree, which is no shorter than the length of the optimal watchman tour. This proves the theorem.

\section{Algorithms for Minimum Guarding Network}

In this section we describe algorithms for finding guarding network, when the communication range of camera nodes is a fixed constant $r>0$. Our aim is to provide practically interesting algorithms for real system implementation, to be explained in the next section. We use two approaches. The first one is to find minimum watchman tour and place cameras along the tour, keep the same visibility coverage. The second one is to find art gallery solutions to ensure visibility coverage and then add extra relay nodes along a spanning tree to connect the guards. We describe the two algorithms respectively and we compare their performance on realistic floor plans.

\section{A. Connected Visibility region Tracking}

In the following we describe the Connected Visibility region Tracking (CVT) algorithm. The first step of CVT is to compute the optimal watchman tour inside a simple polygon. We adapt the algorithm by Chin et al. [5] and Tan et al. [26] which in runtime $O\left(n^{4}\right)$ finds the shortest watchman route for simple polygon through a given point $s$ within the polygon. The concept is to find the "cuts" in $P$ that the watchman route must touch to guard the whole polygon, and visit these cuts using a shortest tour.

For a given polygon $P$, suppose $v_{i}$ is a reflex vertex in $P$ and one of its adjacent vertices is $v^{\prime}$. We shoot a ray from $v^{\prime}$ to $v$, hitting the polygon at $y$, then the visibility cut $C=\overline{v y}$ is a cut of $P$ and separated $P$ into two parts. We call the part of $P$ not containing $v^{\prime}$ the essential piece of $P$, denoted as $P(C)$. Suppose the watchman route has not visited the part of 


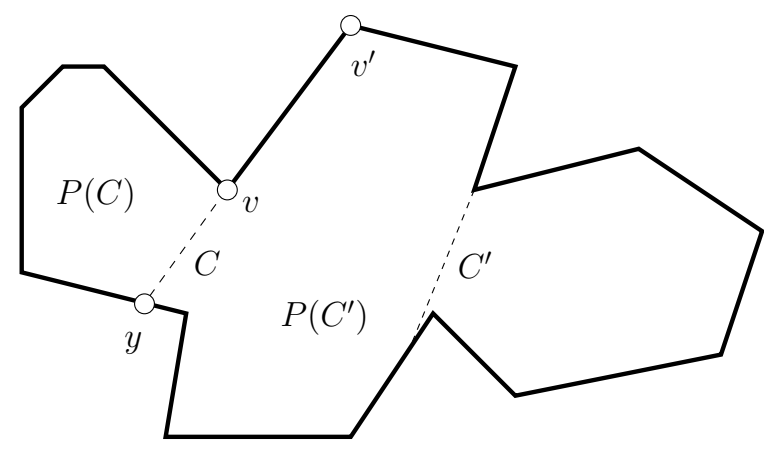

Fig. 2: Cut $C$ dominates $C^{\prime}$ since $P\left(C^{\prime}\right)$ contains $P(C)$ - any tour that visits $P(C)$ must also visit $C^{\prime}$ and guard $P\left(C^{\prime}\right) . C$ is also an essential cut.

$P(C)$ yet, then it must at least touch the visibility cut $C$ in the later route to guard $P(C)$. A visibility cut $C_{j}$ is dominated by another cut $C_{i}$ if $P\left(C_{j}\right)$ contains $P\left(C_{i}\right)$, which means if the route passes $C_{i}$ to touch $P\left(C_{i}\right)$, then $P\left(C_{j}\right)$ is covered. We call a cut essential cut if it is not dominated by any other cuts.

With the essential cut, the origin watchman route problem is reduced to finding the shortest route that touches every essential cut inside a polygon. In brief, we triangulate the given polygon and "reflex" the polygon using the essential cuts as mirrors in order. After these reflections we pick the shortest path connecting the starting point $s$ and its image, which becomes a tour in the original polygon $P$. We elaborate the details below.

We first list the essential cuts in clockwise order, $\left\{C_{1}, C_{2}, \cdots, C_{k}\right\}$. Starting from point $s$, we want to find a path to visit this cut list. We enumerate on the first cut to visit starting from $s$ and take the shortest tour. If the first cut to visit is $C_{i}$, then the tour visits $\pi=C_{i}, C_{i+1}, \cdots, C_{k}, C_{1}, \cdots, C_{i-1}$ in this order.

Once the path touches the next essential cut $C$ on the list, we reflect the polygon using $C$ as an mirror. Thus the tour goes straight through the cut in the reflected copy. In the original polygon $P$ the tour is reflected back at $C$. The path finding process will stop when it visits the last cut $C_{i-1}$ in the list and it goes back to $s$. In other words, the reflections with respect to the cuts will generate a sequence of $k$ copies of the polygon $P$ glued along the cuts in the same order $\pi$ - this glued polygon is denoted by $\hat{P}$. The minimum watchman tour is found by finding the shortest path inside $\hat{P}$ connecting the starting point $s$ and the image of $s$ in the last copy of $P$. We can get the watchman route $T$ by mapping this path back to the original polygon.

Once the shortest watchman route $R$ of a polygon $P$ is acquired, the cameras will be installed at every vertex of $R$. Furthermore, we walk through all the cuts and add cameras at the intersections of $T$ with them only when needed (i.e., if the cameras placed at junctions of $R$ cannot cover $P\left(C_{i}\right)$ for a cut $C_{i}$, we add one more camera at the intersection of $C_{i}$ with $R$ ). This set of guards is sufficient to guard $P$. See figure 3 for example.

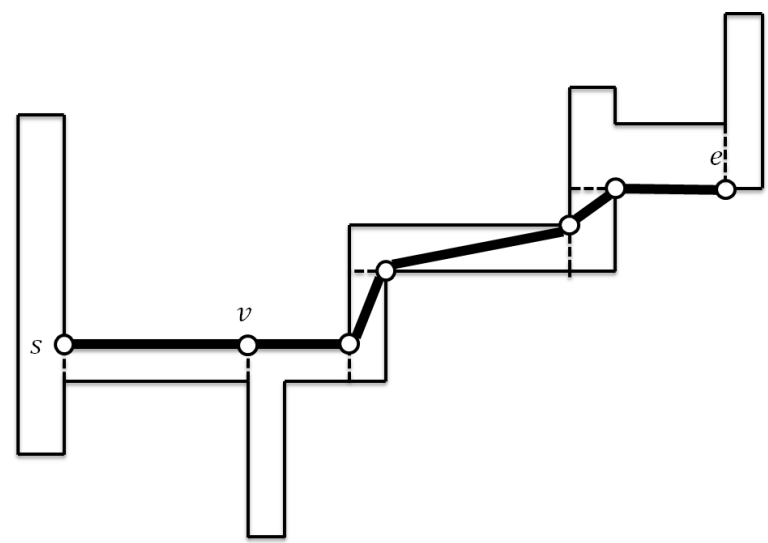

Fig. 3: For a given polygon $P$ with a starting point $s$ with watchman route marked as red line and cameras as red dots. The essential cut $C_{e}$ of $P$ corresponding to $s$ is marked as red dashed line, the visibility cuts of $P$ according to $s$ are marked as green dashed line. Notice that a guard (in green) is added at the intersection of the watchman tour and one visibility cut.

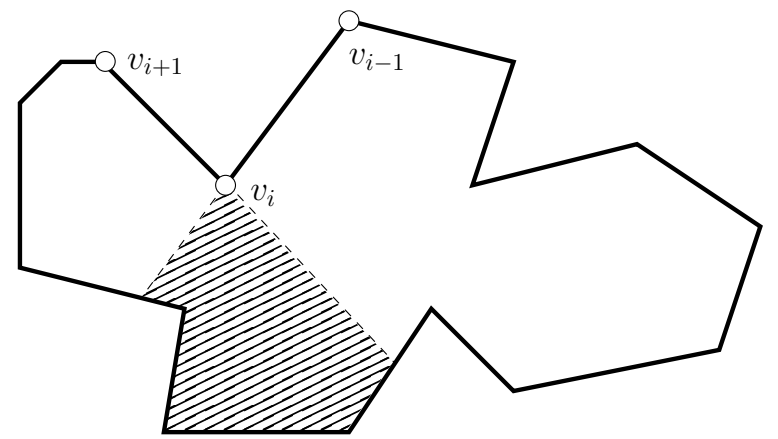

Fig. 4: The guarding region of $v_{i}$ (shaded).

Once the cameras are placed, we add extra relay nodes (with or without cameras) along the watchman tour to connect the adjacent cameras. The relay nodes are placed uniformly while ensuring adjacent relay nodes have distances smaller than communication range $r$.

\section{B. Connected Visibility region Planning}

The second algorithm, Connected Visibility region Planning (CVP), is to first find an art gallery solution to ensure visibility coverage and then add extra relay nodes along a spanning tree to connect the guards. In a simple polygon without holes, only reflex vertices can block the view. The basic idea of CVP is to deploy cameras such that for each reflex point in $P$ that may obstructs the view, at least one camera can look around it.

Let $v_{i}$ be a reflex vertex of $P$, and $v_{i-1}$ and $v_{i+1}$ be its two neighboring vertices, then we define a guarding region $R\left(v_{i}\right)$ as the wedge $W_{i}$ bounded by extending the two edges $v_{i+1} v_{i}$ and $v_{i-1} v_{i}$ inside $P$. We select a guard within $W_{i}$. See Figure 4 for an example.

We choose a hitting set $M=\left\{m_{1}, m_{2}, \cdots, m_{k}\right\}$ of the guarding regions for all reflex vertices, i.e., any guarding region contains at least one guard in $M$.

Finding a hitting set for a collection of geometric regions is NP-hard. But one could use a greedy algorithm to find a 
solution with approximation ratio of $\ln n$ [7]. Specifically, at each stage, we will find a guard that lies in the maximum number of uncovered reflex vertices, until all reflex vertices are covered.

We place cameras at the positions of the guards selected above. We add extra relay nodes to connect them into a connected graph, similar to the case of watchman tour based algorithm. In this case, we use the minimum spanning tree connecting the guards. If any edge in the MST is longer than $r$ (the communication range of these nodes), we deploy relays along this edge uniformly to ensure adjacent relay nodes have distances smaller than $r$.

\section{Relay Deployment}

To perform monitoring tasks, all camera nodes must be connected to the network. Connectivity is achieved when two camera nodes are within each other's communication range. When the distances between adjacent deployment locations of camera nodes in the MST exceed this range, which is possible in many cases, wireless relay nodes are needed. Its main functionality is to exchange data messages among camera nodes. For sparse camera node deployments, more than one relay nodes are deployed to connect adjacent cameras.

The optimization goals of relay nodes deployment are: (1) forming a connected wireless camera network so that every node can reliably transfer their data to a specified base station. (2) minimizing the total communication power consumption.

The key parameter here is the communication range, which is dynamic in real systems because it's affected by the deployment environments. For example, the communication range of a node in an open corridor is longer than that of a node in an office with closed door. For deployment planning CVP, we choose a conservative communication range for all nodes that ensures a connected graph. For dynamic camera deployment CVT, variable communication ranges are used for nodes at different locations. The specific communication range can be obtained at deployment time [18].

If relay nodes are equipped with camera sensors, their visibility coverage after deployment can overlap with that of original guards. Therefore, it provides opportunity to remove the original camera nodes while maintaining full visibility coverage. Intuitively, for any camera $g_{i} \in G$, consider the two, if such exist, relays $g_{j}, g_{k}$ that have the closest distance from $g_{i}$. If $R\left(g_{i}\right) \subseteq R\left(g_{j}\right) \cup R\left(g_{k}\right)$ and distance $\left(g_{j}, g_{k}\right)<r$, then the camera $g_{i}$ can be eliminated. An example is shown in Figure 5.

\section{SYSTEM IMPLEMENTATION}

We implement a wireless camera network testbed for indoor deployment and evaluation. This testbed has 12 batterypowered wireless camera nodes, each node is built based on the off-the-shelf BeagleBone low power development board [8]. It is a credit-card-sized (3.4" $\times 2.1$ ") Linux computer with an AM335x $720 \mathrm{MHz}$ ARM processor. We choose the BeagleBone as our deployment platform because its processor is powerful enough for video processing, but its low power

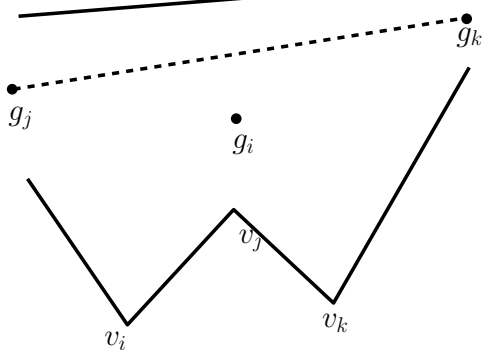

Fig. 5: Guard Reduction

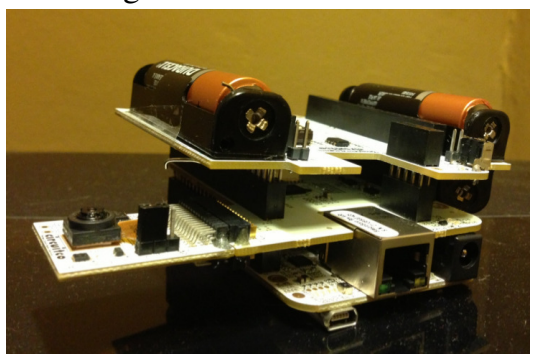

Fig. 6: Battery-powered Wireless Camera Node

design allows it to be powered by 4 AA batteries. Moreover, it has rich hardware interfaces to expand the its functionality, including plug-in components like cameras and wireless radio adapters. A picture of the camera node is shown in Figure 6.

For each camera node, we install the Angstrom distribution [19], which is an Linux operating system distribution designed for low power embedded devices. For Wi-Fi communication, each node utilizes a USB Wi-Fi dongle with Realtek RTL8192CU chipset. We also attempted an alternative setup by installing Ubuntu version 13.04 and Belkin F5D7050v3 USB Wi-Fi dongle. In our observation, there is no discernible difference in the results between these two setups.

Each BeagleBone is equipped with a 3.1 megapixel Aptina CMOS digital image sensor MT9T111 via the extension board. This camera integrates on-chip functions such as anti-shake and auto focus, and is programmable through serial interface. To be applied in face identification applications, the horizontal pixel density requirement is approximately $5 \mathrm{pixel} / \mathrm{cm}$. Therefore, by applying the auto focus function, the maximum scene width is 4 meters, with variable monitoring distance within the building ( $<50$ feet). Major system parameters of the camera are shown in the Table I.

\section{A. Experiments}

We firstly plot the floor plan of the Computer and Information Science department building in Temple University as

\begin{tabular}{|l|c|}
\hline System Parameters & Value \\
\hline \hline Maximum Picture Resolution & 2048 x 1536 \\
\hline Maximum Frame Rate & $30 \mathrm{fps}$ \\
\hline Wireless Communication Range & $60+\mathrm{feet}$ \\
\hline Wireless Bandwidth & $54 \mathrm{Mbps}$ \\
\hline Processing Speed & $720 \mathrm{MHz}$ \\
\hline
\end{tabular}

TABLE I: Camera Node Specification 


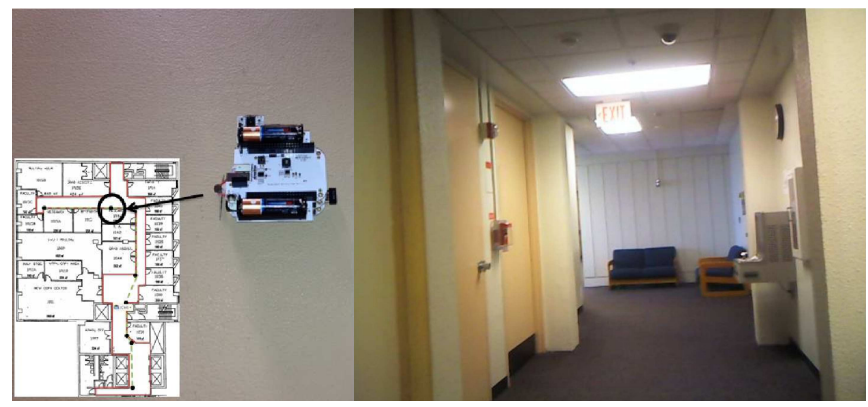

(a) System Deployment

(b) Pictures taken by sensor node

Fig. 8: Sensor Node Deployment

\begin{tabular}{|l|c|c|}
\hline Link ID & Length(feet) & Packet Loss Rate \\
\hline \hline 1 & 25 & $4 \%$ \\
\hline 2 & 8 & $0 \%$ \\
\hline 3 & 30 & $4 \%$ \\
\hline 4 & 15 & $0 \%$ \\
\hline 5 & 43 & $0 \%$ \\
\hline 6 & 13 & $0 \%$ \\
\hline 7 & 44 & $0 \%$ \\
\hline
\end{tabular}

TABLE II: CVP Wireless Link Packet Loss Rate

sample input polygon for deployment algorithms. As shown in Figure 7(a), the thick line represents the input polygon. In Figure 7(b), 7(c), and 7(d), the black points represent camera deployment locations and the dash lines represent the wireless links. The actual camera system deployed on the wall is shown in Figure 8.

Next we evaluate the network's wireless connectivity. We set the Wi-Fi mode to be Ad-Hoc, and tune $2.412 \mathrm{Ghz}$ as the communication frequency. The power management function is turned off so that the Wi-Fi communication will be running at highest performance.

For each wireless link in this network deployed in Figure 7b, we execute the "ping" command between each pair of nodes for 50 times and record the packet loss rate. The result is shown in Table II. We have assigned each wireless link an ID. We can see the lengths of the wireless links range from 8 to 44 feet, and all links have packet loss ratios smaller than $5 \%$.

To assess the wireless connection quality, we conducted a long term experiment. We deploy two cameras at the two ends of the corridor, which is 43 feet in length. We ping from one of the node to the other once per second for one hours and record the success rate. This experiment is repeated for three different times. The results are shown in Table III.

It is apparent from Table III that reliable wireless connec-

\begin{tabular}{|c|c|c|c|c|}
\hline Exp & \# Transmitted & \# Received & Avg RTT & Duration \\
\hline \hline 1 & 3580 & 3573 & $16.875 m s$ & $3593893 \mathrm{~ms}$ \\
\hline 2 & 3909 & 3901 & $18.007 m s$ & $3927517 \mathrm{~ms}$ \\
\hline 3 & 3693 & 3689 & $35.513 m s$ & $3722935 \mathrm{~ms}$ \\
\hline
\end{tabular}

TABLE III: Long Term Wireless Connectivity tions are ensured. The packet loss rates are all smaller than $1 \%$. Since the length of the wireless link is 43 feet, which is the longest one in the CVP deployment in our department building floor plan, we can see that the CVP deployment forms a reliable wireless network with no need for extra wireless relay nodes.

Finally we test the system's power by measuring the voltage and current when the Wi-Fi module is both on and off. We found that the power of a node is about $1.22 \mathrm{~W}$ and $0.72 \mathrm{~W}$ when the Wi-Fi is on and off, respectively. An ordinary AA Alkaline long-life battery can hold about 5000J of energy, so the system is expected to sustain for hours. We configure a node to take pictures and exchange hello messages with its neighbours, and its battery life is above 5 hours.

\section{B. Discussion}

Since we adopt visibility sensing and circular communication models in our deployment algorithms, it is important to validate these models with real experiments. We have tested qualities of sensing and communication using our testbed in real deployment experiments. Experimental results show that both models are valid. However, for effective and efficient camera network deployment, both models can be further improved under realistic constraints.

Visibility Model. Line of sight is a simplified abstraction for optical sensing. In real deployment, various factors, including indoor obstacles and light settings, influence the sensing quality. Building constructions, such as interior doors and furnitures can block the view of cameras. Solution to this problem is to adaptively adjust the height of camera deployments produced by deployment algorithm. In our experiments, most of the obstacles can be avoided after in-situ tuning during the deployment. Light settings can also significantly affect the quality of pictures taken by the optical sensor. In our building, although the lights are always on, the level of illumination is not consistent in different areas. To obtain clear views of all the areas, extra camera nodes may be needed to cover relatively dark areas.

Wireless Communication Model. The realistic communication range is not a perfect disk. Instead, it is highly directional under a complex indoor building structure like our department. The communication range of a node in the corridor is much larger than that of a node in the office room. This observation suggests that relay nodes' positions can be fine tuned during deployment. Another issue is that the Wi-Fi channel may need to be tuned at deployment time, to avoid interference with existing wireless networks if any are presented.

From these observations, we can see that it is also important to perform in-situ tuning on sensing and communication after deployment planning.

\section{Simulation}

\section{A. Experiment Setup}

We have implemented a simulation framework for the camera deployment algorithms. In the experiments, we take 


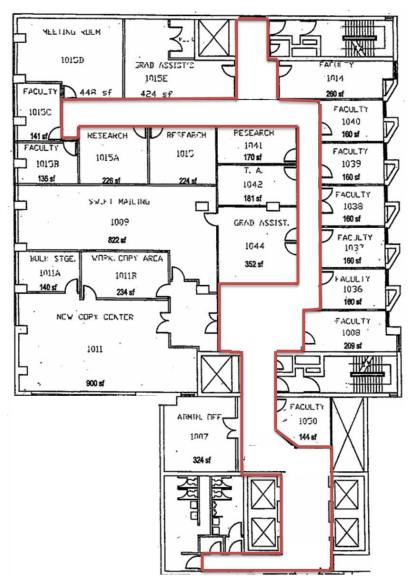

(a) Floor Plan

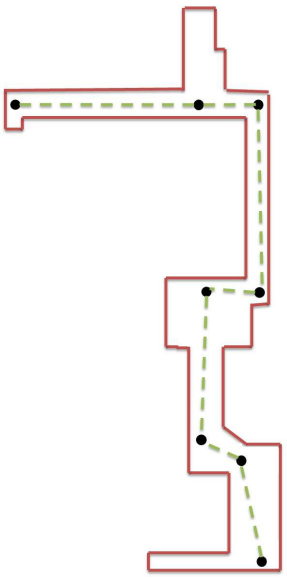

(b) CVP Deployment

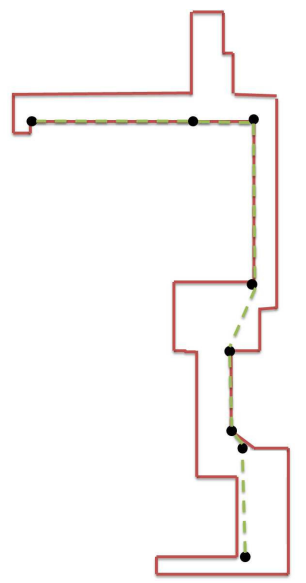

(c) CVT Deployment

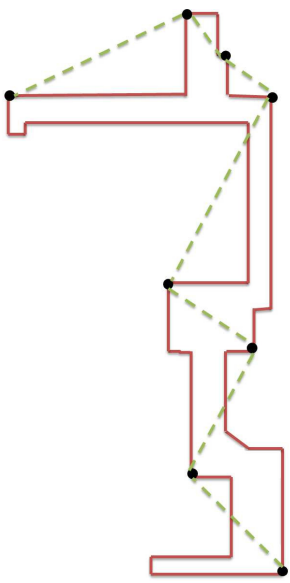

(d) 3-Coloring Deployment

Fig. 7: Simulation with a Real Floor Plan

the floor plans of 20 realistic buildings as inputs. These buildings include hotels, classrooms, houses and museums. The CVP, CVT and 3-coloring algorithm are implemented. The simulation shows that all three algorithms are able to ensure full visibility coverage, so we will focus on comparing their camera numbers, number of relay nodes and wireless transmission power.

As a baseline, we choose the well known 3-coloring algorithm [30]. It firstly triangulate the polygon $P$. The vertices of the polygon are then 3-colored in such a way that every triangle has all three different colors. Once a 3-coloring is found, the color with the fewest vertices forms a valid guard set with at most $\lfloor n / 3\rfloor$ guards [10].

\section{B. Camera Number}

The camera number is an important metric to evaluate the performance of a deployment algorithm. The camera number is directly related to the construction cost. Besides, as the camera number grows, the video data size also increases. This will cast heavier burden on power supply because wireless data communication is energy expensive. To evaluate the algorithms' performance, we simulate them on 20 real floor plans and record the required camera numbers. The results are shown in Figure 9.

Figure 9 compares CVP, CVT and 3-coloring algorithm in terms of camera numbers. They require 4.2, 4.8 and 5.9 cameras on average, respectively. Besides, the 3-coloring algorithm's camera number has a standard deviation of 2.1, while those of CVP and CVT are 1.6. Therefore, on average, CVP and CVT require fewer cameras than the 3-coloring algorithm.

\section{Number of Relay Nodes}

To assess these three algorithms in terms of wireless connectivity, we compare the number relay nodes required by them. Since the wireless communication quality depends on many factors, which cannot be predicted before in-situ experiments,

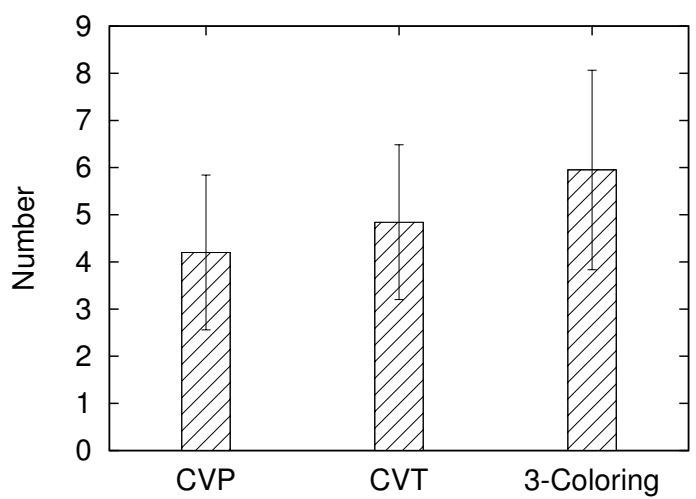

Fig. 9: Camera Number

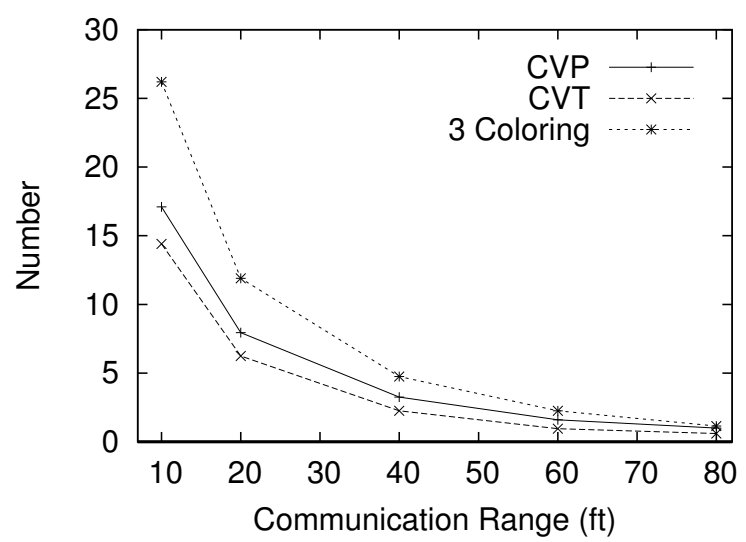

Fig. 10: Number of Relay Nodes

we conduct simulations with various different communication ranges. Figure 10 presents the necessary number of relay nodes required by these three algorithms.

From Figure 10, we can see that the numbers of relay nodes drop dramatically when the communication range increases from 10 to 40 feet, but remains relatively stable when this value grows greater than 40 feet. These results indicate that 
TABLE IV: Radio Characteristics

\begin{tabular}{|l|c|}
\hline Operation & Energy Dissipated \\
\hline \hline Transmitter Electronics $\left(E_{T x-e l e c}\right)$ & \\
Receiver Electronics $\left(E_{R x-e l e c}\right)$ & $50 \mathrm{~nJ} / \mathrm{bit}$ \\
$\left(E_{T x-e l e c}=E_{R x-e l e c}=E_{\text {elec }}\right)$ & \\
\hline Transmit Amplifier $\left(\epsilon_{a m p}\right)$ & $100 \mathrm{pJ} / \mathrm{bit} / \mathrm{m}^{2}$ \\
\hline
\end{tabular}

when designing realistic systems, the communication range should be around 40 feet, on average, so that maximum marginal benefits are improved.

We also discover that the CVT has a good performance in terms of relay node number. On average, it requires 14.4, 6.25, $2.25,0.95$ and 0.6 relays when the communication range is 10 , 20, 40, 60 and 80 feet, respectively. This is because the CVT deploys cameras along the shortest watchman route, which is the shortest path that can guard the polygon. Since the CVT's total distance of wireless links is smaller, it requires less relays when compared with CVP and 3-coloring algorithm.

It is noted that using the CVP and CVT algorithms, the wireless links are mostly in direct line of sight, which greatly facilitate the deployment of wireless relays. Besides, when two camera nodes are in direct line of sight, their mutual communication range is larger compared to the condition when obstacles exist because obstacles significantly affect wireless communication quality. In experiment, we can see that using 3-Coloring deployment, obstacles between connected cameras frequently occur.

\section{Transmission Cost}

The wireless transmission cost is another important issue in the wireless camera network, because the battery capacity is limited and the power consumption of wireless transmission is large. Currently, there is a great deal of research in the area of radio transmission cost in wireless sensor networks. In this paper, we will adopt the radio model constructed in [11]. To transmit $k$ bits of message to a distance $d$ using this radio model, the power consume by a node is shown in Equation 1 .

$$
\begin{array}{r}
E_{T x}(k, d)=E_{T x-\text { elec }}(k)+E_{T x-a m p}(k, d) \\
E_{T x}(k, d)=E_{\text {elec }} * k+\epsilon_{\text {amp }} * k * d^{2}
\end{array}
$$

To receive this message, the power spends by wireless node is shown in Equation 2:

$$
\begin{array}{r}
E_{R x}(k)=E_{R x-\text { elec }}(k) \\
E_{R x}(k)=E_{\text {elec }} * k
\end{array}
$$

The physical meaning of the parameters in Equation 1 and 2 are summarized in Table IV.

The camera nodes are modeled after the VIVOTEK CC8130 1MP Panoramic View camera. they are operating at frame rate of $10 \mathrm{fps}$ with image resolution at $1280 \times 800$. The images are encoded in H.264 format and the video will be compressed with a ratio of $30 \%$. Therefore, the data bandwidth of each camera is $254 \mathrm{kbit} / \mathrm{s}$. If the camera network keeps operating

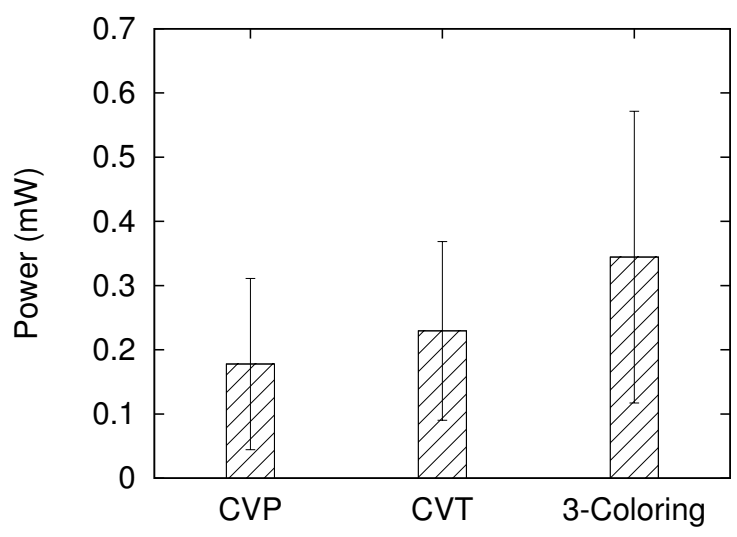

Fig. 11: Communication Power

for 5 hours, then each camera node will generate $4.5 \mathrm{Gbit}$ of data.

In order to collect these video data, the camera nodes send them link after link to arrive at a data sink. In this experiment, we select one camera node for each camera network to be the data sink, and compute power consumption of the network during the data transmission process.

From Figure 11, we can see that using CVP, CVT and 3coloring algorithm to deploy camera networks, the network communication power is $0.18,0.23$ and $0.34 \mathrm{~mW}$, respectively. One reason why CVP achieves lower communication cost than 3 -coloring is that CVP deploys cameras in the internal regions of the polygons, while the 3-coloring algorithm deploys cameras at the vertices of the polygons. On average, deploying cameras in the internal regions can reduce the mutual distances between the camera nodes. Since the wireless power consumption is proportional to the square of link distance, reducing camera nodes' mutual distances can effectively reduce the communication power.

In summary, CVP and CVT outperform the classical 3coloring algorithm in terms of camera number, number of relay nodes and communication power. Specifically, CVP can achieve a near optimal performance in necessary camera numbers, while CVT can reduce the number of relays significantly. Both these algorithms can reduce communication power compared with 3-coloring algorithm.

\section{RELATED WORK}

The Art Gallery problem, finding the minimum number of guards to see a given polygon, is a classical problem in computational geometry and is well known to be NP-hard [24]. It is also well known that $\lfloor n / 3\rfloor$ cameras are occasionally necessary and always sufficient to cover a simple polygon with $n$ vertices [6]. In a polygon with $n$ vertices and $h$ holes, $\lfloor(n+h) / 3\rfloor$ vertex guards are always sufficient [13]. If the polygon is orthogonal (having only horizontal and vertical edges), $\lfloor n / 4\rfloor$ vertex guards are always sufficient [16].

The Watchman Tour Problem deals with finding a route in a simple polygon $P$ such that each point in the interior of $P$ can be seen from at least one point along the route[28]. [4] 
proposes an $O\left(n^{4}\right)$ algorithm to solve the problem of finding the shortest watchman route that is restricted to pass through a starting point $s$ on the boundary of $P$. [23] gave an $O\left(f(n) n^{2}\right)$ time solution to the problem without restricting to any starting points. The authors of [1] applies the concept of essential cut to solve this problem. They design an algorithm to find out all essential cuts in the polygon, then a simple route that visits all these essential cuts will ensure coverage of the entire polygon.

To solve the camera network deployment problem, previous researchers proposed many different camera models and deployment algorithms. [22] "cone" shape of camera views. The authors of [33] construct a detailed model for the 3dimensional field of view of cameras. [31] introduces a camera deployment scenario that requires that neighbouring cameras should have hand-off regions. [21] models obstacles in a probabilistic way, and designs a deployment algorithm that ensures visibility coverage with high confidence. However, all these papers only focus on visibility coverage, and none of these papers consider about wireless connectivity. Instead, in our paper we assume the camera's sensing range is only restricted by line of sight, and we optimize the communication costs while ensuring visibility coverage.

\section{CONCLUSION}

We formally define the Connected Minimum Guarding Set problem. We prove the hardness of the problem and design 2approximation algorithm in the geometric setting. Inspired by solutions to watchman tour and art gallery problem, we also develop two algorithms to solve this problem. Experiments are conducted on an implemented prototype of the proposed system to evaluate its feasibility and the effectiveness. In extended simulations, we test the proposed algorithms on realistic floor plans. All the experiment results demonstrate that the proposed algorithms can ensure visibility coverage and reduce camera numbers and communication power significantly.

Acknowledgments Huang, Shneider and Lin would like to acknowledge support by NSF through CNS-1239108, NSF CNS-1218718 and NSF IIS-1231680. Ni, Ban and Gao would like to acknowledge support by NSF through NSF DMS-1221339, NSF CNS-1217823 and NSF CNS-1116881.

\section{REFERENCES}

[1] S. Carlsson, H. Jonsson, and B. J. Nilsson. Finding the shortest watchman route in a simple polygon. Discrete \& Computational Geometry, 22(3):377-402, 1999.

[2] W. Chin and S. Ntafos. Optimum watchman routes. In 2nd Proc. ACM Symposium on Computational Geometry, pages 24-33, New York, New York, USA, 1986. ACM Press.

[3] W. Chin and S. Ntafos. Optimum watchman routes. Inform. Process. Lett., 28:39-44, 1988.

[4] W.-p. Chin and S. Ntafos. Optimum watchman routes. Information Processing Letters, 28(1):39-44, 1988.

[5] W. P. Chin and S. Ntafos. Shortest watchman routes in simple polygons. Discrete \& Computational Geometry, 6(1):9-31, 1991.

[6] V. Chvátal. A combinatorial theorem in plane geometry. J. Combin. Theory Ser. B, 18:39-41, 1975.

[7] V. Chvatal. A greedy heuristic for the set-covering problem. Mathematics of operations research, 4(3):233-235, 1979.

[8] G. Coley. BeagleBone Rev A3 System Reference Manual. BeagleBoard.org, 2011.
[9] C. K. Cowan and P. D. Kovesi. Automatic sensor placement from vision task requirements. Pattern Analysis and Machine Intelligence, IEEE Transactions on, 10(3):407-416, 1988.

[10] S. Fisk. A short proof of chvátal's watchman theorem. Journal of Combinatorial Theory, Series B, 24(3):374, 1978.

[11] W. R. Heinzelman, A. Chandrakasan, and H. Balakrishnan. Energyefficient communication protocol for wireless microsensor networks. In System Sciences, 2000. Proceedings of the 33rd Annual Hawail International Conference on, pages 10-pp. IEEE, 2000.

[12] F. Hoffmann, M. Kaufmann, and K. Kriegel. The art gallery theorem for polygons with holes. In Foundations of Computer Science, 1991. Proceedings., 32nd Annual Symposium on, pages 39-48. IEEE, 1991.

[13] F. Hoffmann, M. Kaufmann, and K. Kriegel. The art gallery theorem for polygons with holes. In Proc. 32nd Annu. IEEE Sympos. Found. Comput. Sci., pages 39-48, 1991.

[14] A. Howard, M. J. Matarić, and G. S. Sukhatme. Mobile sensor network deployment using potential fields: A distributed, scalable solution to the area coverage problem. In Distributed Autonomous Robotic Systems 5 , pages 299-308. Springer, 2002.

[15] C.-F. Huang and Y.-C. Tseng. The coverage problem in a wireless sensor network. Mobile Networks and Applications, 10(4):519-528, 2005.

[16] J. Kahn, M. M. Klawe, and D. Kleitman. Traditional galleries require fewer watchmen. SIAM J. Algebraic Discrete Methods, 4:194-206, 1983.

[17] D. Lee and A. Lin. Computational complexity of art gallery problems. Information Theory, IEEE Transactions on, 32(2):276-282, 1986.

[18] H. Liu, J. Li, Z. Xie, S. Lin, K. Whitehouse, J. A. Stankovic, and D. Siu. Automatic and robust breadcrumb system deployment for indoor firefighter applications. In Proceedings of the 8th international conference on Mobile systems, applications, and services, pages 21-34. ACM, 2010.

[19] M. Luca and K. Kooi. Angstrom Manual. Embedded Power, 2010.

[20] C. S. Mata and J. S. B. Mitchell. Approximation algorithms for geometric tour and network design problems (extended abstract). In Proceedings of the eleventh апnual symposium on Computational geometry, SCG '95, pages 360-369, New York, NY, USA, 1995. ACM.

[21] A. Mittal and L. S. Davis. Visibility analysis and sensor planning in dynamic environments. In Computer Vision-ECCV 2004, pages 175189. Springer, 2004.

[22] A. T. Murray, K. Kim, J. W. Davis, R. Machiraju, and R. Parent. Coverage optimization to support security monitoring. Computers, Environment and Urban Systems, 31(2):133-147, 2007.

[23] B. J. Nilsson and S. Carlsson. Guarding art galleries: Methods for mobile guards. 1994

[24] J. O'rourke. Art gallery theorems and algorithms, volume 1092. Oxford University Press Oxford, 1987.

[25] S. Ram, K. Ramakrishnan, P. Atrey, V. Singh, and M. Kankanhalli. A design methodology for selection and placement of sensors in multimedia surveillance systems. In Proceedings of the 4th ACM international workshop on Video surveillance and sensor networks, pages 121-130. ACM, 2006.

[26] X. TAN. Corrigendum to an incremental algorithm for constructing shortest watchman routes. Int. J. Comput. Geom. Appl., 9:319-323, 1999.

[27] X. Tan. Fast computation of shortest watchman routes in simple polygons. Information Processing Letters, 77(1):27-33, 2001.

[28] X. Tan. Fast computation of shortest watchman routes in simple polygons. Information Processing Letters, 77(1):27-33, 2001.

[29] X. Tan, T. Hirata, and Y. Inagaki. An incremental algorithm for constructing shortest watchman routes. International Journal of Computational Geometry \& Applications, 3(04):351-365, 1993.

[30] J. Urrutia. Art gallery and illumination problems. Handbook of computational geometry, pages 973-1027, 2000.

[31] Y. Yao, C.-H. Chen, B. Abidi, D. Page, A. Koschan, and M. Abidi. Sensor planning for automated and persistent object tracking with multiple cameras. In Computer Vision and Pattern Recognition, 2008. CVPR 2008. IEEE Conference on, pages 1-8. IEEE, 2008.

[32] Y. Yao, C.-H. Chen, B. Abidi, D. Page, A. Koschan, and M. Abidi. Can you see me now? sensor positioning for automated and persistent surveillance. Systems, Man, and Cybernetics, Part B: Cybernetics, IEEE Transactions on, 40(1):101-115, 2010.

[33] J. Zhao, S. Cheung, et al. Multi-camera surveillance with visual tagging and generic camera placement. In Distributed Smart Cameras, 2007. ICDSC'07. First ACM/IEEE International Conference on, pages 259266. IEEE, 2007. 